\title{
THE DUAL FORM OF THE APPROXIMATION PROPERTY FOR A BANACH SPACE AND A SUBSPACE
}

\author{
T. FIGIEL AND W. B. JOHNSON*
}

\begin{abstract}
Given a Banach space $X$ and a subspace $Y$, the pair $(X, Y)$ is said to have the approximation property (AP) provided there is a net of finite rank bounded linear operators on $X$ all of which leave the subspace $Y$ invariant such that the net converges uniformly on compact subsets of $X$ to the identity operator. The main result is an easy to apply dual formulation of this property. Applications are given to three space properties; in particular, if $X$ has the approximation property and its subspace $Y$ is $\mathcal{L}_{\infty}$, then $X / Y$ has the approximation property.
\end{abstract}

In memory of A. Pełczyński

\section{InTRODUCTION}

In [FJP] the authors and the late A. Pełczyński introduced the notion of the bounded approximation property (BAP) for a Banach space $X$ and a subspace $Y$. The pair $(X, Y)$ is said to have the approximation property (AP) provided the identity on $X$ is the $\tau$-limit of a net of finite rank bounded linear operators on $X$ all of which leave the subspace $Y$ invariant. Here we recall that the $\tau$-topology on the space $L(X)$ of bounded linear operators on the Banach space $X$ is the topology of uniform convergence on compact subsets of $X$. If the approximating net of finite rank operators can be chosen so that their norms are uniformly bounded by $\lambda$, then $(X, Y)$ is said to have the $\lambda$-bounded approximation property $(\lambda$-BAP), and $(X, Y)$ has the BAP provided it has the $\lambda$-BAP for some $\lambda<\infty$. When the subspace $Y$ is either the whole space or the zero subspace, these concepts reduce to the classical concepts of AP and BAP for a single space. Obviously if $(X, Y)$ has the BAP then $(X, Y)$ has the AP. Non obvious is the fact, pointed out by Lissitsin and Oja [LO, Corollary 5.12], that if $X$ is reflexive and $(X, Y)$ has the AP, then $(X, Y)$ has the 1-BAP. The root for this is

Date: August 4, 2015.

1991 Mathematics Subject Classification. Primary 46B20, 46B28.

* Supported in part by NSF DMS-1310550. 
Grothendieck's classical theorem $\mathrm{Gr}$, that the AP implies the 1-BAP for reflexive spaces, as improved by Godefroy-Saphar GS1, Theorem 1.5], Oja and collaborators (see, e.g., [LMO] and references therein), and others. The AP for a pair $(X, Y)$ was not considered in [FJP], but even if one cares only about reflexive spaces it is probably worthwhile to consider the concept, in part because the dual form of the AP is simply stated and easy to work with while the dual form of the BAP is more problematical. In this note we prove in Theorem 2.1 the dual form for what it means for a pair $(X, Y)$ to have the $\mathrm{AP}$ and give a couple of applications. In a paper under preparation we will give a far reaching extension of the duality result where the subspace $Y$ is replaced by a nest of subspaces of $X$. Although the proof of the general result is not essentially more complicated than what is treated here, it does require introducing concepts extraneous to the context of this short note. It seemed to us that the special case considered here as well as the applications were interesting enough to warrant a separate publication. The applications of Theorem 2.1 are new approximation property three space results in the spirit of other such results (see e.g. GS2 and [CK]).

We use standard Banach space theory notation and concepts, as are contained e.g. in [LT].

\section{JoINT AP}

We begin with a special case of a known lemma (part (1) is contained in [R2] and part (2) is in [Sp]) but include a simple proof.

Lemma 1. Let $\mathcal{F}_{Y}(X)=\{T \in \mathcal{F}(X): T Y \subseteq Y\}$.

1. $x^{*} \otimes x \in \mathcal{F}_{Y}(X)$ if and only if either $x^{*} \in Y^{\perp}$ or $x \in Y$.

2. If $F \in \mathcal{F}_{Y}(X)$, then $F$ is the sum of $n$ rank one elements of $\mathcal{F}_{Y}(X)$, where $n$ is the rank of $F$.

Proof: For (1), if $x^{*} \in Y^{\perp}$ then $\left(x^{*} \otimes x\right) Y=0$ so $x^{*} \otimes x \in \mathcal{F}_{Y}(X)$. If $x \in Y$ then $\left(x^{*} \otimes x\right) X \subseteq \operatorname{span}\{x\} \subseteq Y$ so $x^{*} \otimes x \in \mathcal{F}_{Y}(X)$. This gives " $\Leftarrow$ ". On the other hand, if $x^{*} \notin Y^{\perp}$ and $x \notin Y$, then there is $y \in Y$ such that $\left\langle x^{*}, y\right\rangle \neq 0$, hence $\left(x^{*} \otimes x\right) y=\left\langle x^{*}, y\right\rangle x \notin Y$, whence $x^{*} \otimes x \notin \mathcal{F}_{Y}(X)$. This gives " $\Rightarrow$ ".

For (2), let $x_{1}, \ldots, x_{m}$ be a basis for $F X \cap Y$ and extend this to a basis for $F X$ by adding $x_{m+1}, \ldots, x_{n}$, so that

$$
\text { span } x_{m+1}, \ldots, x_{n} \cap Y=\{0\} .
$$

Write $F=\sum_{k=1}^{n} x_{k}^{*} \otimes x_{k}$. By part (1) of this lemma, $x_{k}^{*} \otimes x_{k} \in \mathcal{F}_{Y}(X)$ for $k \leq m$. To complete the proof it is by (1) sufficient to show that for all $k>m$ we have $x_{k}^{*} \in Y^{\perp}$. If for some $k>m$ we had $x_{k}^{*} \notin Y^{\perp}$, 
then, choosing $y \in Y$ with $\left\langle x_{k}^{*}, y\right\rangle \neq 0$, we would have by (*) that $\sum_{j=m+1}^{n}\left\langle x_{j}^{*}, y\right\rangle x_{j} \notin Y$. But $\sum_{j=1}^{m}\left\langle x_{j}^{*}, y\right\rangle x_{j} \in Y$, so we would have Fy $\notin Y$, a contradiction.

Theorem 2.1 is the main result of this note. $N(X, Z)$ denotes the nuclear operators from $X$ to $Z$ and is abbreviated as $N(X)$ when $X=$ $Z$. In the hypothesis we assume that the space $X$ has the AP in order to formulate the theorem with nuclear operators $N(X)$ rather than with the projective tensor product of $X^{*}$ with $X$. Given $T \in N(X)$, $\operatorname{tr}(T)$ is the trace of $T$, which is well-defined when $X$ has the AP by Grothendieck's fundamental result [Gr], [LT, Theorem 1.e.15].

Theorem 2.1. Suppose that $Y \subseteq X$ and $X$ has the AP. The following are equivalent.

1. The pair $(X, Y)$ has the $A P$.

2. For all $T \in N(X)$ for which $T X \subseteq Y$ and $T Y=0$ we have $\operatorname{tr}(T)=0$.

\section{Proof:}

$(1) \Longrightarrow(2)$. Assume (2) is false and get $T \in N(X)$ so that $T Y=0$ and $T X \subseteq Y$ but $\operatorname{tr}(T)=1$. So $T \in L(X, \tau)^{*}$ and $\langle I, T\rangle=1$. Let $F \in \mathcal{F}_{Y}(X)$. We want to show that $\langle F, T\rangle=0$, which would contradict (1). By Lemma 1, it is enough to check that $\left\langle x^{*} \otimes x, T\right\rangle=0$ if either $x^{*} \in Y^{\perp}$ or $x \in Y$. But $\left\langle x^{*} \otimes x, T\right\rangle=\left\langle x^{*}, T x\right\rangle$, so this is clear from the facts that $T x \in Y$ and $T Y=0$.

$(2) \Longrightarrow(1)$. If (1) is false and $X$ has the AP, we can separate $I$ from $\mathcal{F}_{Y}(X)$ with a $\tau$ continuous linear functional on $L(X)$, which, since $X$ has the AP, is represented by a nuclear operator $T$ on $X$ (see [Gr], [LT, Theorems 1.e.3, 1.e.4]). Then $\operatorname{tr}(T)=\langle I, T\rangle \neq 0$ but $\langle F, T\rangle=0$ for all $F \in \mathcal{F}_{Y}(X)$. In particular, $\left\langle x^{*}, T x\right\rangle=\left\langle x^{*} \otimes x, T\right\rangle=0$ if either $x^{*} \in Y^{\perp}$ or $x \in Y$. So if $x \in X$, then for all $x^{*} \in Y^{\perp}$ we have $\left\langle x^{*}, T x\right\rangle=0$, which is to say that $T x \in\left(Y^{\perp}\right)_{\perp}=Y$. So $T X \subseteq Y$. If $y \in Y$, then for all $x^{*} \in X^{*}$ we have $\left\langle x^{*}, T y\right\rangle=\left\langle x^{*} \otimes y, T\right\rangle=0$, which says that $T Y=0$.

A sequence $Y \rightarrow X \rightarrow Z$ of Banach spaces is a short exact sequence (ses) when the operator $Y \rightarrow X$ is an isomorphic embedding and the operator $X \rightarrow Z$ is surjective and has $Y$ as its kernel. Up to passing to equivalent norms, this is just saying that $Y$ is a subspace of $X$ and $Z$ is the quotient space $X / Y$. A ses $Y \rightarrow X \rightarrow Z$ locally splits if the dual ses $Z^{*} \rightarrow X^{*} \rightarrow Y^{*}$ splits, which just means that $Z^{*}$ is a complemented subspace of $X^{*}$. This is equivalent to saying that finite dimensional subspaces of $Z$ uniformly lift to $X$. The theory of ses of Banach spaces 
is presented in $\mathrm{CG}$, but much more than we use is contained in $[\mathrm{J}$, Corollary 1.4] and the discussion preceding that Corollary. If $X \rightarrow X^{* *}$ is the natural embedding, then the ses $X \rightarrow X^{* *} \rightarrow X^{* *} / X$ locally splits, but $X \rightarrow X^{* *} \rightarrow X^{* *} / X$ need not split (e.g., $X=c_{0}$ ).

Proposition 1. Suppose that $Y \rightarrow X \rightarrow X / Y$ is a short exact sequence that locally splits, and $Y^{* * *}$ and $X$ both have the AP. Then the pair $(X, Y)$ has the $A P$ and hence $X / Y$ has the $A P$.

Proof: By Theorem 2.1 it is enough to show that if $T$ is a nuclear operator on $X$ such that $T X \subseteq Y$ and $T Y=0$, then the trace of $T$ is zero. Consider $T$ as an operator into $X^{* *}=Y^{\perp \perp} \oplus Z$ (where $Z$ is isomorphic to $\left.(X / Y)^{* *}\right)$. This is also nuclear, and composing with the projection of $X^{* *}$ onto $Y^{\perp \perp}\left(\equiv Y^{* *}\right)$ we see that $T$ is also nuclear when considered as an operator into $Y^{* *}$. Since $Y^{* * *}$ has the AP, by the corrected theorem of Grothendieck $\mathrm{Gr}$ proved by Oja and Reinov OR, $T$ is nuclear when considered as an operator from $X$ into $Y$. Since $T$ is zero on $Y$, the trace of $T$ is zero.

Remark. The assumption on $Y$ in Proposition 1 cannot be weakened to " $Y^{* *}$ has the AP". (Consider a James-Lindenstrauss $Y$ such that $Y^{* *}$ has a basis and $Y^{* *} / Y$ is a reflexive space that fails the AP, and let $X=Y^{* *}$. It was this kind of example that led Oja and Reinov to the correct statement of Grothendieck's "theorem".)

Corollary 1. Suppose that $Y \rightarrow X \rightarrow X / Y$ is a short exact sequence, $X$ has the $A P$, and $Y$ is $\mathcal{L}_{\infty}$. Then the pair $(X, Y)$ has the $A P$ and hence $X / Y$ has the $A P$.

Proof: The short exact sequence locally splits because $Y$ is $\mathcal{L}_{\infty}$. The space $Y^{* * *}$ is $\mathcal{L}_{1}$ and thus has the AP, so the conclusion follows from Proposition 1 .

In Corollary 1, the roles of $X$ and $Y$ can be interchanged.

Corollary 2. Suppose that $Y \rightarrow X \rightarrow X / Y$ is a short exact sequence, $X$ is $\mathcal{L}_{\infty}$, and $Y$ has the $A P$. Then the pair $(X, Y)$ has the $A P$ and hence $X / Y$ has the $A P$.

Proof: By Theorem 2.1 it is enough to show that if $T$ is a nuclear operator on $X$ such that $T X \subseteq Y$ and $T Y=0$, then the trace of $T$ is zero. Just as in Proposition 1, for that it is enough to check that $T$ is nuclear when considered as an operator into $Y$. It is, by an observation of Stegall and Retherford [SR, Theorem III.3], because $X$ is $\mathcal{L}_{\infty}$.

Remark. The BAP version of Corollary 2 was proved in [FJP]. 
Next we prove a BAP version of Corollary 1, It gives a slight improvement of the Castillo-Moreno [CM, Lemma 3.1] result that $X / Y$ has the BAP if $X$ has the BAP and $Y$ is $\mathcal{L}_{\infty}$. Our direct geometrical argument gives an alternate proof of the Castillo-Moreno result but is clumsier than the algebraic argument in [CM] or the proof above of Corollary 1 ,

Proposition 2. Suppose that $Y \rightarrow X \stackrel{Q}{\rightarrow} X / Y$ is a short exact sequence, $X$ has the $B A P$, and $Y$ is $\mathcal{L}_{\infty}$. Then the pair $(X, Y)$ has the $B A P$.

Proof: Let $G$ be a finite dimensional subspace of $X$. We want to find $T \in \mathcal{F}_{Y}(X)$ that is the identity on $G$ and has "good" norm (here and in the following "good" means that the norm is independent of $G$ ). Since $Y$ is $\mathcal{L}_{\infty}$, the short exact sequence locally splits, so there is an operator $U: Q G \rightarrow X$ with $Q U$ the identity on $Q G$ and $\|U\|$ depends only on how well the short exact sequence locally splits. So $Y+U Q G$ is a "good" direct sum decomposition of the space $Y+U Q G$ because $U$ is a "good" isomorphism on $Q G$ and $Q U$ is the identity on the range of $Q G$. By basic linear algebra, there is a finite dimensional subspace $E$ of $Y$ such that $G \subseteq E+U Q G$. Now $X$ has the BAP, so there is $S \in \mathcal{F}(X)$ with $S$ the identity on $U Q G$ and the norm of $S$ controlled by the BAP constant of $X$.

We next replace $S$ with an operator $S_{1} \in \mathcal{F}(X)$ that is still the identity on $U Q G$, has controlled norm, and is zero on $Y$ (so that $S_{1} \in$ $\left.\mathcal{F}_{Y}(X)\right)$. Since $G$ is arbitrary and $U Q G=Q G$, this will give the Castillo-Moreno lemma mentioned above. To get $S_{1}$, we define an operator $V \in \mathcal{F}(X)$ that agrees with $S$ on $Y$ with $S V$ vanishing on $U Q G$ so that $\|V\|$ is controlled and set $S_{1}:=S-V$. The $\mathcal{L}_{\infty}$ structure of $Y$ is used to define $V$. Write $Y$ as a directed union of a net $Y_{\alpha}$ of subspaces of $Y$ so that the $Y_{\alpha}$ are uniformly isomorphic to $\ell_{\infty}^{n_{\alpha}}$; $n_{\alpha}<\infty$. Since $Y+U Q G$ is a "good" direct sum decomposition, the projections $P_{\alpha}$ from $Y_{\alpha}+U Q G$ onto $Y_{\alpha}$ that are zero on $U Q G$ have uniformly bounded norm, and, by the injective property of $\ell_{\infty}$ spaces, these projections extend to uniformly bounded projections (still denoted by $\left.P_{\alpha}\right)$ from $X$ onto $Y_{\alpha}$. Of course, the net $\left(P_{\alpha}\right)$ converges pointwise on $Y$ to the identity on $Y$, so the net $S P_{\alpha}$ has (since $S$ has finite rank) a subnet that converges pointwise on $X$, necessarily to a finite rank operator $V$ that agrees with $S$ on $Y$ and is zero on $U Q G$. This completes the construction of $S_{1}:=S-V$ (and, incidentally, our alternate proof for [CM, Lemma 3.1]). The remainder of the proof is very easy. Just take $\alpha$ so that $E$ is a subspace of $Y_{\alpha}$ and define $T:=P_{\alpha}+S_{1}$. 


\section{REFERENCES}

[CG] J. M. F. Castillo and M. Gonzalez, Three-space problems in Banach space theory. Lecture Notes in Mathematics, 1667, Springer-Verlag, Berlin (1997), $\mathrm{xii}+267 \mathrm{pp}$.

[CM] J. M. F. Castillo and Y. Moreno, On the bounded approximation property in Banach spaces, Israel J. Math. 198 (2013), no. 1, 243-259.

[CK] C. Choi and J. M. Kim, On dual and three space problems for the compact approximation property, J. Math. Anal. Appl. 323 (2006), no. 1, 78-87

[FJP] T. Figiel, W. B. Johnson, and A. Pełczyński, Some approximation properties of Banach spaces and Banach lattices, Israel J. Math. 183 (2011), 199-231.

[GS1] G. Godefroy and P. D. Saphar, Duality in spaces of operators and smooth norms on Banach spaces, Illinois J. Math. 32 (1988), no. 4, 672-695.

[GS2] G. Godefroy and P. D. Saphar, Three-space problems for the approximation properties, Proc. Amer. Math. Soc. 105 (1989), no. 1, 70-75.

[Gr] A. Grothendieck, Produits tensoriels topologiques et espaces nuclaires, Mem. Amer. Math. Soc. 16 (1955), no. 16, 140 pp.

[J] W. B. Johnson, Extensions of c $c_{0}$, Positivity 1 (1997), no. 1, 55-74.

[LT] J. Lindenstrauss and L. Tzafriri, Classical Banach Spaces, Vol I, Ergebn. Math. Grenzgeb. 92, Springer, 1977.

[LMO] A. Lissitsin, K. Mikkor, and E. Oja, Approximation properties defined by spaces of operators and approximability in operator topologies, Illinois J. Math. $\mathbf{5 2}$ (2008), no. 2, 563-582.

[LO] A. Lissitsin and E. Oja, The convex approximation property of Banach spaces, J. Math. Anal. Appl. 379 (2011). 616-626.

[OR] E. Oja and Reinov, A counterexample to A. Grothendieck, Eesti NSV Tead. Akad. Toimetised Fs.-Mat. 37 (1988), no. 1, 14-17, 121.

[R2] J. R. Ringrose, On some algebras of operators, Proc. London Math. Soc. (3) 15 (1965), 61-83.

[Sp] N. K. Spanoudakis, Generalizations of certain nest algebra results., Proc. Amer. Math. Soc. 115 (1992), no. 3, 711-723.

[SR] C. P. Stegall and J. R. Retherford, Fully nuclear and completely nuclear operators with applications to $\mathcal{L}_{1}$ - and $\mathcal{L}_{\infty}$-spaces, Trans. Amer. Math. Soc. 163 (1972), 457-492.

Institute of Mathematics, The Polish Academy of Sciences

E-mail address: t.figiel@impan.gda.pl

Department of Mathematics, Texas A\&M University, College StaTION, TX 77843-3368 U.S.A

E-mail address: johnson@math.tamu.edu 